$12.1 ; 15.2$

\title{
Радиоактивационный контроль плотности износостойких покрытий AIN и CrN на кремнии
}

\author{
(C) В.А. Рыжков, В.А. Тарбоков, Е.А. Смолянский, Г.Е. Ремнев \\ Инженерная школа новых производственных технологий, Томский политехнический университет, Томск, Россия \\ E-mail: ryzhkov@tpu.ru
}

Поступило в Редакцию 1 февраля 2021 г.

В окончательной редакции 12 февраля 2021 г.

Принято к публикации 19 фревраля 2021 г.

\begin{abstract}
С помощью комбинации двух методик (неразрушающего радиоактивационного анализа на пучках протонов и оптической микроинтерферометрии) измерены соответственно массовые и линейные толщины покрытий AlN и $\mathrm{CrN}$, осажденных на кремниевые подложки методом магнетронного распыления. Показано, что при линейных толщинах от 2.2 до $5.7 \mu \mathrm{m}$ плотность покрытий близка к значениям для объемных материалов $\operatorname{AlN}\left(3.26 \mathrm{~g} / \mathrm{cm}^{3}\right)$ и $\mathrm{CrN}\left(5.9 \mathrm{~g} / \mathrm{cm}^{3}\right)$, а стехиометрию нитридов можно контролировать, изменяя параметры магнетронного осаждения. Методика может быть использована также для определения плотности покрытий из карбидов и оксидов металлов, которые применяются в качестве износостойких покрытий.
\end{abstract}

Ключевые слова: плотность, радиоактивационный анализ, магнетрон, микроинтерферометр.

DOI: 10.21883/PJTF.2021.10.50969.18722

Необходимость использования методов неразрушающего контроля плотности покрытий представляется несомненной. Для определения плотности тонких металлических $(\mathrm{Cu}, \mathrm{Nb}, \mathrm{Mo}$, Та и $\mathrm{W})$ и углеродных алмазоподобных покрытий, осажденных из абляционной плазмы, генерированной в результате воздействия мощных импульсных ионных пучков на поверхность соответствующих металлов и графита, нами была использована комбинация методов оптической эллипсометрии с рентгенофлуоресцентным анализом (РФA, XRF) при возбуждении радиоактивным источником ${ }^{109} \mathrm{Cd}$ и регистрации характеристического рентгеновского излучения металлов $\mathrm{Si}(\mathrm{Li})$-детектором, а для алмазоподобных пленок - комбинация методик интерферометрии с радиоактивационным анализом на пучках дейтронов циклотрона $\mathrm{У}-120 \mathrm{M}$ по реакции ${ }^{12} \mathrm{C}(d, n){ }^{13} \mathrm{~N}[1]$. В этом случае для расчета массовой толщины применялись тонкие образцы сравнения с известной массовой толщиной. В работе [2] проведено сравнение методов для определения толщины осажденных PVD-методом (PVD - physical vapor deposition) покрытий алюминия для получения упаковочных изделий. В [3] для нахождения массовых толщин покрытий, осажденных на подложки, использован метод РФА, основанный на детектировании кремниевым дрейфовым детектором (silicon drift detector, $\mathrm{SSD})$ характеристического излучения как элементов покрытия, так и подложки под разными углами обзора, что в принципе не требует использования образцов сравнения. В работе [4] была сделана попытка обойти проблему использования в методе РФА сертифицированных образцов сравнения при применении для расчетов моделирования методом Монте-Карло. В целом рассмотренные выше подходы [2-4] к определению толщин покрытий без использования образцов сравнения выглядят ненадежными и неоправданными, поскольку выбор или изготовление тонких образцов сравнения материалов с известной массовой толщиной определяемых элементов не представляет серьезной проблемы.

Цель настоящей работы - тестирование радиоактивационной методики определения массовой толщины покрытий нитридов, карбидов и оксидов с толщиной до нескольких микрометров.

Для определения плотности покрытий $\mathrm{AlN}$ и $\mathrm{CrN}$, ocaждаемых магнетроном на подложки кремния, массовая толщина покрытий контролировалась радиоактивационным и РФА-методами с использованием тонких образцов сравнения, а линейные толщины покрытий определялись с помощью микроинтерферометра на образцахсвидетелях, устанавливаемых рядом с исследуемыми образцами в процессе осаждения. Кремниевая подложка выбрана в качестве модельной ввиду того, что из нее при облучении протонами в выбранном интервале энергий не образуются радионуклиды с периодами полураспада более нескольких секунд, что позволяет проводить неразрушающее радиоактивационное определение элементов покрытий, включая и возможные загрязнения углеродом и кислородом. Кроме того, характеристическое рентгеновское излучение $K$-серии кремния примерно в одинаковой степени поглощается тонкими покрытиями $\mathrm{AlN}$ и $\mathrm{CrN}$, что было использовано для дополнительного контроля массовой толщины исследуемых покрытий РФА-методом. При использовании подложек из других элементов могут возникать помехи при измерении наведенной активности позитронных излучателей ${ }^{11} \mathrm{C}$, ${ }^{13} \mathrm{~N}$ и ${ }^{18} \mathrm{~F}$. В таких случаях может быть использован альтернативный подход, описанный нами в работе [1], радиоактивационное определение массовых толщин по- 
Результаты определения плотности покрытий

\begin{tabular}{c|c|c|c|c|c|c|c|c}
\hline Тип & $\begin{array}{c}T_{\text {sub }}, \\
{ }^{\circ} \mathrm{C}\end{array}$ & $\begin{array}{c}\sigma_{f}, \\
\mathrm{GPa}\end{array}$ & $\begin{array}{c}\delta, \\
\mu \mathrm{m}\end{array}$ & $\begin{array}{c}X(\mathrm{~N}), \\
\mathrm{mg} / \mathrm{cm}^{2}\end{array}$ & $\begin{array}{c}X(M e), \\
\mathrm{mg} / \mathrm{cm}^{2}\end{array}$ & $\begin{array}{c}X(M e \mathrm{~N}), \\
\mathrm{mg} / \mathrm{cm}^{2}\end{array}$ & $\begin{array}{c}\mathrm{N} / M e, \\
\text { atom/atom }\end{array}$ & $\begin{array}{c}d, \\
\mathrm{~g} / \mathrm{cm}^{3}\end{array}$ \\
\hline AlN-1 & 280 & -0.1 & 4.6 & 0.50 & $(0.96)$ & 1.46 & $(1)$ & 3.17 \\
AlN-2 & 120 & +0.1 & 5.7 & 0.64 & $(1.23)$ & 1.87 & $(1)$ & 3.28 \\
AlN-3 & 500 & -0.8 & 4.2 & 0.435 & $(0.84)$ & 1.275 & 1.31 & 0.78 \\
CrN-5 & 300 & -0.3 & 2.2 & 0.23 & 1.08 & 1.53 & 0.97 & 5.95 \\
CrN-6 & 300 & +0.2 & 3.1 & 0.32 & 1.21 & 1.44 & 0.74 & 6.55 \\
CrN-7 & 300 & -1.1 & 2.2 & 0.24 & 1.2 & &
\end{tabular}

крытий на основе $\mathrm{C}, \mathrm{N}$ и $\mathrm{O}$ с активацией дейтронами с энергией около $3 \mathrm{MeV}$, когда устраняется активация средних и тяжелых элементов, массовую толщину которых в случае необходимости определяют РФА-методом.

Для отработки режимов осаждения покрытий с различным уровнем внутренних напряжений на кремниевые подложки были нанесены покрытия AIN (толщиной 4-6 $\mu \mathrm{m})$ и $\mathrm{CrN}(2-3 \mu \mathrm{m})$ при следующих параметрах магнетронного напыления: мощность магнетрона $1.5 \mathrm{~kW}$ $(\mathrm{AlN})$ и $1.0 \mathrm{~kW}(\mathrm{CrN})$, парциальное давление реакционных газов $1.2 \cdot 10^{-3}$ Torr, расстояние от подложки до мишени $150 \mathrm{~mm}$. При формировании $\mathrm{AlN}$-покрытий варьировалась температура подложки $T_{s u b}$ (см. таблицу) при постоянном соотношении напускаемых в рабочую камеру газов $\mathrm{Ar} / \mathrm{N}_{2}$, равном 10:4 для всех образцов. При получении покрытий $\mathrm{CrN}$ соотношение $\mathrm{Ar} / \mathrm{N}_{2}$ соответствовало 1:1 для образцов CrN-5 и CrN-7 и 3:1 для образца $\mathrm{CrN}-6$ (обозначения образцов соответствуют указанным таблице). В случае образца $\mathrm{CrN}-7$ использовано отрицательное смещение напряжения $100 \mathrm{~V}$.

РФА-спектрометр ARL QUANT'X (Thermo Fisher Scientific, USA) с анодом из родия был использован в качестве вспомогательного инструмента, особенно в случае покрытий AlN, для которых протонная активация не позволяла определить массовую толщину алюминия. В случае покрытий AlN контролировалась степень пропускания ими излучения $K$-серии кремния (подложки), a в случае покрытий $\mathrm{CrN}$ дополнительно контролировалась интенсивность пика $K_{\alpha}$-серии хрома. В таблице в колонке $X(M e)$ значения массовой толщины алюминия в покрытиях AlN оценены РФА-методом в предположении стехиометричности данных покрытий, а также исходя из значений массовой толщины азота, определенной радиоактивационным методом (эти значения приведены в скобках).

Для радиоактивационного определения толщины покрытий нитридов $\mathrm{AlN}$ и $\mathrm{CrN}$ на $\mathrm{Si}$-подложках использовано одновременное облучение образцов в течение $20 \mathrm{~min}$ расфокусированным пучком протонов циклотрона У-120М (Томский политехнический университет) с начальной энергией $6.8 \mathrm{MeV}$, выведенным через титановую фольгу толщиной $40 \mu \mathrm{m}\left(18 \mathrm{mg} / \mathrm{cm}^{2}\right)$ на воздух, что снижало энергию протонов до $6.1 \mathrm{MeV}$. Шесть пластин кремния размером $4 \times 1 \times 0.1 \mathrm{~cm}$ с покрытиями из $\mathrm{AlN}$ и $\mathrm{CrN}$ были установлены на вращающемся мишенедержателе вместе с двумя образцами сравнения того же поперечного сечения $\left(4 \mathrm{~cm}^{2}\right)$, что обеспечивало равенство числа бомбардирующих протонов на каждом образце. В качестве образца сравнения массовых толщин азота, углерода и кислорода была использована пленка капрона (Nylon-6) с формулой [-HN $\left.\left(\mathrm{CH}_{2}\right)_{5} \mathrm{CO}-\right]_{n}$ толщиной $5 \mu \mathrm{m}$, a в качестве образца сравнения массовой толщины хрома - слой $\mathrm{Cr}$ с массовой толщиной $1.1 \mathrm{mg} / \mathrm{cm}^{2}$, электрохимически осажденный на медную фольгу толщиной $20 \mu \mathrm{m}$. Общий ток протонов на держателе контролировался в пределах $1 \mu \mathrm{A}$, что ограничивало плотность тока на образцах величиной $15 \mathrm{nA} / \mathrm{cm}^{2}$. Образцы были плотно упакованы в алюминиевую фольгу толщиной $14 \mu \mathrm{m}$, использованную в качестве контейнера, предохраняющего образцы от возможного загрязнения примесями из окружающей атмосферы во время облучения за счет внедрения ядер отдачи из реакций на элементах окружающей атмосферы (C, N, O), а также в качестве индикатора такого загрязнения. Наведенная активность в этих фольгах была несколько выше фоновой, поэтому возможным вкладом ядер радионуклидов, внедрившихся из атмосферы в исследуемые слои, пренебрегалось. Кроме того, перед упакованными образцами устанавливалась алюминиевая фольга толщиной $100 \mu \mathrm{m}$ для снижения энергии протонов в среднем до $4.33 \mathrm{MeV}$ и значительного расширения их распределения по энергии (за счет страгглинга и неравномерности толщины фольги) для того, чтобы минимизировать погрешности, связанные с наличием узких резонансов в эффективных сечениях ядерных реакций, используемых для анализа, в диапазоне энергии протонов от 2 до $6 \mathrm{MeV}$. Покрытие AlN толщиной около $5 \mu \mathrm{m}$ снижает энергию протонов примерно до $4 \mathrm{MeV}$, что сушественно выше порогов ядерных реакций ${ }^{14} \mathrm{~N}(p, \alpha){ }^{11} \mathrm{C} \quad\left(E_{t h r}=3.13 \mathrm{MeV}\right),{ }^{13} \mathrm{C}(p, n){ }^{13} \mathrm{~N}$ $\left(E_{t h r}=3.235 \mathrm{MeV}\right)$ и ${ }^{18} \mathrm{O}(p, n){ }^{18} \mathrm{~F}\left(E_{t h r}=2.57 \mathrm{MeV}\right), \mathrm{c}$ помощью которых контролировалось массовое содержание $\left(\right.$ в $\left.\mathrm{mg} / \mathrm{cm}^{2}\right)$ азота, углерода и кислорода в покрытиях по числу ядер ${ }^{11} \mathrm{C}\left(T_{1 / 2}=20.38 \mathrm{~min}, E_{\gamma}=511 \mathrm{keV}\right)$, ${ }^{13} \mathrm{~N} \quad(9.965 \mathrm{~min}, 511 \mathrm{keV})$ и ${ }^{18} \mathrm{~F}$ (109.78 min, $\left.511 \mathrm{keV}\right)$ соответственно.

Для образцов с покрытием из нитрида хрома по реакциям ${ }^{54} \mathrm{Cr}(p, n){ }^{54} \mathrm{Mn} \quad\left(E_{t h r}=2.2 \mathrm{MeV}, 312.3\right.$ days, $834.8 \mathrm{keV}),{ }^{50} \mathrm{Cr}(p, \alpha){ }^{47} \mathrm{~V} \quad\left(E_{t h r}=3.46 \mathrm{MeV}, 32.6 \mathrm{~min}\right.$, 


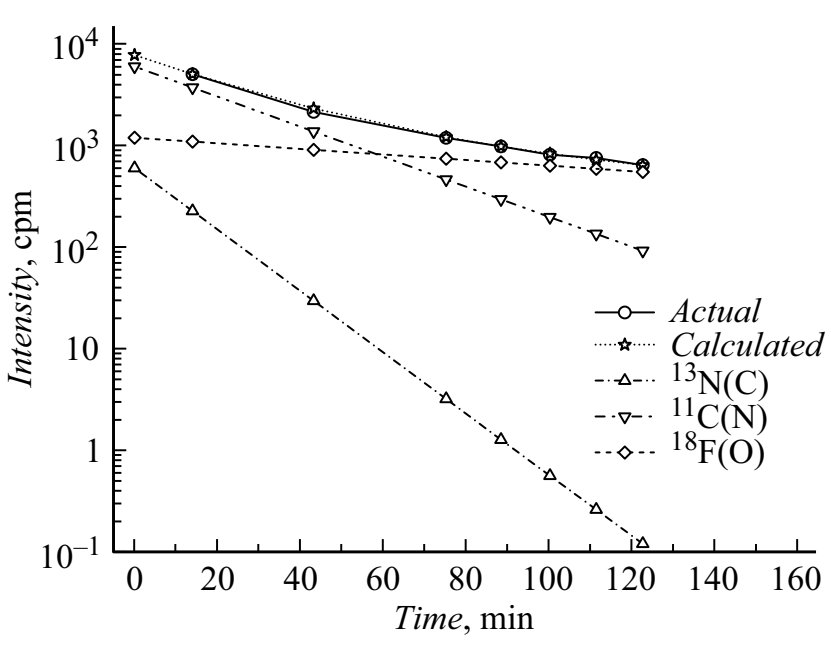

Кривая распада (в полулогарифмическом масштабе) и ее компоненты в образце AlN-1.

$511 \mathrm{keV})$ и ${ }^{52} \mathrm{Cr}(p, n){ }^{52,52 m} \mathrm{Mn}\left(E_{t h r}=5.6 \mathrm{MeV}, 5.59\right.$ days и $21.1 \mathrm{~min}$; 744.2, 935.5 и $1434 \mathrm{keV}$ ) контролировалось также массовое содержание хрома (в $\left.\mathrm{mg} / \mathrm{cm}^{2}\right)$. Радионуклиды, образуемые из кремния и алюминия, имеют периоды полураспада не более нескольких секунд, а потому полностью распадались уже через $1-2 \mathrm{~min}$, тогда как первое измерение коаксиальным детектором из высокочистого германия (Canberra) в свинцовой защите начиналось через $15 \mathrm{~min}$ после облучения. Наведенная активность шести исследуемых образцов и двух образцов сравнения измерялась последовательно (по $60 \mathrm{~s}$ для каждого образца), после чего цикл измерений повторялся еще семь раз, чтобы выявить возможные примеси углерода (по $\left.{ }^{13} \mathrm{~N}\right)$ и кислорода (по ${ }^{18} \mathrm{~F}$ ) для чистых позитронных излучателей, кроме аналитического радионуклида ${ }^{11} \mathrm{C}$. Типичная кривая распада позитронной активности, наведенной в образце, и ее компоненты для образца AlN-1 приведены на рисунке, где „Actual ${ }^{6}$ значения измеренной активности, а „Calculated ${ }^{\text {" }}$ рассчитанной по сумме выделенных компонент методом наименьших квадратов.

Активность ${ }^{13} \mathrm{~N}$, наводимая из примеси углерода, вносит наименьший вклад в активность образцов, тогда как ${ }^{18} \mathrm{~F}$, образуемый из кислорода, определяет активность образцов к концу измерений кривой распада - через $2 \mathrm{~h}$ после начала измерений. Кислород во всех образцах, судя по соотношению активностей аналитических радионуклидов, вероятнее всего, был локализован не на поверхности пленок нитридов, а на межфазной границе между пленками нитридов и кремниевыми подложками (в виде пленок оксидов металлов и/или кремния). Массовую толщину пленок нитридов алюминия $X(M e \mathrm{~N})$, где $M e=\mathrm{Al}$, рассчитывали по активности радионуклида ${ }^{11} \mathrm{C}$, считая эти покрытия стехиометрическими, поскольку оценки массовой толщины алюминия РФА-методом в пределах погрешности измерения соответствовали массовым толщинам азота, определенным радиоактива- ционным методом. Массовая толщина пленок нитридов хрома $X(M e \mathrm{~N})$, где $M e=\mathrm{Cr}$, рассчитывалась по активности радионуклидов ${ }^{11} \mathrm{C}$ для массовой толщины азота и ${ }^{54} \mathrm{Mn},{ }^{47} \mathrm{~V}$ и ${ }^{52,52 m} \mathrm{Mn}$ для массовой толщины хрома. С помощью оптического микроинтерферометра МИИ-4 (АО „ЛОМО“, Санкт-Петербург) были определены линейные толщины покрытий $\delta$ на образцах-свидетелях с погрешностью $\pm 0.1 \mu \mathrm{m}$. Величина механических напряжений $\sigma_{f}$ в структуре пленка-подложка оценивалась по формуле Стоуни [5]. Отношение массовых $X(M e \mathrm{~N})$ и линейных толщин $\delta$ покрытий позволило получить их плотности $d$, приведенные вместе с остальными контролируемыми параметрами в таблице.

Из таблицы видно, что плотность трех покрытий AIN $\left(3.16 \pm 0.12 \mathrm{~g} / \mathrm{cm}^{3}\right)$ воспроизводится относительно среднего значения с намного меньшим отклонением $( \pm 4 \%)$, чем плотность трех покрытий $\mathrm{CrN}-5.81 \pm 0.81 \mathrm{~g} / \mathrm{cm}^{3}$ $( \pm 14 \%)$, что может быть следствием исключения погрешности определения массовой толщины металла (Al), поскольку покрытия AlN считались стехиометрическими. Наиболее стехиометрическое покрытие нитрида хрома ( $\mathrm{CrN}-6)$ неожиданно обнаружило наименьшую плотность, что вряд ли может быть обусловлено тем, что средние внутренние напряжения в этом образце были наиболее растягивающими $(+0.2 \mathrm{GPa})$.

Таким образом, в работе показано, что при линейных толщинах от 2.2 до $5.7 \mu$ m плотность покрытий близка к значениям для объемных материалов $\mathrm{AlN}\left(3.26 \mathrm{~g} / \mathrm{cm}^{3}\right)$ и $\mathrm{CrN}\left(5.9 \mathrm{~g} / \mathrm{cm}^{3}\right)$, а стехиометрию нитридов можно контролировать, изменяя параметры магнетронного осаждения. Также показана возможность радиоактивационного контроля загрязнения получаемых магнетронным осаждением структур углеродом и кислородом. Данная радиоактивационная методика может быть использована и для определения плотности износостойких покрытий карбидов и/или оксидов металлов.

\section{Финансирование работы}

Исследование выполнено при финансовой поддержке Российского фонда фундаментальных исследований и Госкорпорации „Росатом“ в рамках научного проекта № 20-21-00025/20.

\section{Конфоликт интересов}

Авторы заявляют, что у них нет конфликта интересов.

\section{Список литературы}

[1] G.E. Remnev, I.F. Isakov, M.S. Opekunov, V.M. Matvienko, V.A. Ryzhkov, V.K. Struts, I.I. Grushin, A.N. Zakoutayev, A.V. Potyomkin, V.A. Tarbokov, A.N. Pushkaryov, V.L. Kutuzov, M.Yu. Ovsyannikov, Surf. Coat. Techol., 114, 206 (1999). DOI: 10.1016/S0257-8972(99)00058-4 
[2] M. Lindner, F. Höflsauer, J. Heider, M. Reinelt, H.-C. Langowski, Thin Solid Films, 666, 6 (2018). DOI: $10.1016 /$ j.tsf.2018.09.032

[3] C. Fiorini, A. Gianoncelli, A. Longoni, F. Zaraga, $X$-Ray Spectrometry, 31, 92 (2002). DOI: 10.1002/xrs.550

[4] W. Giurlani, E. Berretti, M. Innocenti, A. Lavacchi, Coatings, 9, 79 (2019). DOI: 10.3390/coatings9020079

[5] G.G. Stoney, Proc. Roy. Soc. Lond. A, 82, 172 (1909). DOI: $10.1098 /$ rspa.1909.0021 